\title{
Asthma COPD Overlap Syndrome: New Phenotype of Obstructive Airway Diseases. Study from Valley of Kashmir
}

\author{
Mir Intikhab ${ }^{1}$, Mohamad Muzzafer Mir², Ursilla Taranum ${ }^{3}$, Sonaullah Shah ${ }^{4}$ \\ ${ }^{1}$ Senior Resident, Internal Medicine, SKIMS, ${ }^{2}$ Senior Resident, Internal Medicine, SKIMS, ${ }^{3}$ Senior Resident, Department of \\ Gynecology and Obstetrics, Government Medical College, Srinagar
}

Corresponding author: Mohamad Muzzafer Mir, MD, C 13 Doctors Hostel, Karanagar, Srinagar, Jammu, Kashmir, India

DOI: http://dx.doi.org/10.21276/ijcmsr.2019.4.1.17

How to cite this article: Mir Intikhab, Mohamad Muzzafer Mir, Ursilla Taranum, Sonaullah Shah. Asthma COPD overlap syndrome: new phenotype of obstructive airway diseases. study from valley of Kashmir. International Journal of Contemporary Medicine Surgery and Radiology. 2019;4(1):A69-A73.

\section{A B S T R A C T}

Introduction: Asthma and chronic obstructive pulmonary disease (COPD) are common diseases with a huge public fitness impact. The aim of this study was to investigate the prevalence of asthma COPD overlap syndrome (ACOS) amongst patients of obstructive airway disease and also to determine the clinical characteristics and demographic profile of such cases with overlap syndrome.

Material and Methods: A hospital based, prospective, observational study was conducted on individuals of both sexes within the age group of 40 and 80 years. Patients with history suggestive of obstructive airway disease were subjected to pulmonary function test. Patients found to have obstructive pattern on pulmonary function test were subjected to sputum eosinophil count and serum IgE levels.

Results: Of 130 patients enrolled in the study 59 (45.38\%) were pure COPD, 48 (36.92\%) were pure Asthma and 23 (17.69\%) were ACOS phenotype. Also patients with ACOS phenotype were found to have more number of acute exacerbations and hospitalizations. Obstructive airway disorders encompass bronchial asthma and chronic obstructive pulmonary disease (COPD).

Conclusion: A subset of patients with obstructive airway disease display characteristics of both asthma and COPD. Such patients are called having asthma COPD overlap syndrome (ACOS). But, the prevalence and the characteristics of these patients with ACOS are not nicely understood.

Keywords: Asthma, COPD Overlap Syndrome, Obstructive Airway Diseases

\section{INTRODUCTION}

It is not yet clear whether COPD and asthma are separate disease entities or they represent the different expressions of the same disorder. ${ }^{1}$ The pathological features, clinical features and response to medications vary between the patients of asthma and COPD. In asthma s patients, there is a predominant Th2 cytokine response and the inflammatory infiltrate consisting predominantly of CD4+cells and eosinophils and the response to steroids is better. On the other hand COPD patients have a predominant Th1 cytokine response and the inflammatory infiltrate consists predominantly of CD8+cells and neutrophils. ${ }^{2,3}$ For several years, evidence based recommendations for asthma and $\mathrm{COPD}^{4,5,6}$ have guided practitioners on the proper assessment and management of these sicknesses. But, the global scientific practice has definitely established the tendencies of the two disorders to overlap despite a different pathophysiological mechanism.Despite the clinical heterogenity, pathophysiological mechanism and response to medications between asthma and COPD,multiple phenotypes of both these diseases have been elucidated. ${ }^{7-13}$ Phenotypic charactrization of both these illnesses and their overlap provides provides a better information regarding the underlying sickness, to define the variations in the analysis and the likely response to the treatment options. ${ }^{8,9}$ Lately, the spanish society of pulmonology and thoracic surgery identified the phenotypic variations by publishing the recommendation which contain COPD phenotypes and suggest individualized treatment approaches depending upon the phenotypic profile. ${ }^{10-12,14-16}$ The most important of these COPD phenotypes is the phenotype that possesses the characteristics of asthma as well as COPD, the combined phenotype(ACOS).ACOS phenotype these days is diagnosed on the basis of the consensus between the Global initative for COPD (GOLD) and Global initative for athma (GINA). ${ }^{17}$ Despite the fact that no universally accepted definition of ACOS exists, available records can assist to discoverthe features of bothv COPD and asthma in this phenotype. This ACOS phenotype has not been extensively studied but there are evidences that support the fact that ACOS patients have an exceotional response to ordinary treatment and this phenotype has a significant public health impact. Phenotypic characterization of both those illnesses and their overlap provides a better information of the basic sickness with the general purpose to define different approaches in 
analysis and responses to different interventions. ${ }^{8,9}$ Lately, the Spanish Society of Pulmonology and Thoracic Surgery (SEPAR) has identified the above concept by publishing recommendations which contain COPD phenotypes and suggest individualized treatment approaches depending on phenotypic profiles. ${ }^{10-12,14-16}$ Based totally on present-day estimates, the most important of these COPD phenotypes represents a population that has features and traits of both COPD and bronchial asthma; the combined phenotype (asthma-COPD overlap). This entity has additionally these days been diagnosed by way of a joint committee among the Global initiative for the Chronic Obstructive Lung disorder (GOLD) and the Global Initiative for Asthma (GINA) and is described as asthma- COPD overlap syndrome (ACOS). ${ }^{17}$ Both COPD and asthma are chronic disorders of the respiratory tract, having features of bronchial obstruction. COPD patients have prominent obstruction and is not reversible. Asthma is characterized by airway hyperresponsiveness and airflow obstruction, which either totally or partly reverses spontaneously or by using medication. COPD commonly presents after the age of 40 , is related to smoking and usually gives airflow reversibility that is not good sized. Bronchial asthma generally manifests earlier, has no association with smoking, has variable symptoms and the obstruction is normally reversible. But the actual situation is extra complicated because we regularly see cases with features of overlap. Some asthma patients smoke and have characteristics similar to COPD, have an accelerated decline in lung function and decreased response to bronchodilators or to corticosteroids. Additionally, we see COPD patients with characteristic features of asthma consisting of reversibility after management with bronchodilators. The cases in whom the features of both the phenotypes overlap could have distinct therapeutic response.

The main goal of our study is to investigate the prevalence of asthma-COPD overlap syndrome among patients with obstructive airway disorder in Kashmiri population. Additionally, we also wanted to study the clinical and demographic profile of patients with asthma-COPD overlap syndrome.

\section{MATERIAL AND METHODS}

In a hospital based study, patients were enrolled within the outpatient branch. Patients of suspected obstructive airway disease within the age group of 40-80 years were subjected to spirometry testing. Patients who had been determined to have an obstructive pattern on spirometry (pre-bronchodilator FEV1 / FVC < 0.7) were enrolled in this study. All the patients enrolled in this study were subjected to detailed history and physical examination. In addition all the enrolled patients were subjected to sputum eosinophils, serum $\mathrm{IgE}$ levels, and $\mathrm{CBC}$ investigations. The data was recorded in a database.

Participants visited the hospital thrice within 30 days. Preand post-bronchodilator spirometry, symptoms, smoking status, medical history and medication use were assessed at visit 1 and sputum induction was performed. At visit 2, venipuncture with blood collection and a skin allergy test ${ }^{18}$ were performed.

\section{Participant Questionnaires}

A detailed history was taken from the patients about cough, sputum production, wheeze, breathlessness, number of hospitalizations during the past 1 year and smoking, Patients included in the study were $>40$ years and $<80$ years of age, in whom prebronchodilator $\mathrm{FEV} 1 / \mathrm{FVC}<0.7$ on spirometry and history suggestive of obstructive airway disease. Airway reversibility and severity of airflow obstruction had been determined by the usage of spirometry. Airway reversibility (bronchodilator responsiveness [BDR]) became defined as an increase in post-bronchodilator FEV1 of $12 \%$ compared to pre-bronchodilator FEV1. Lower respiratory sputum portions had been selected from saliva and processed using dithiothreitol, and differential counts have been calculated. ${ }^{19}$ Asthma is defined as episodic respiratory signs and completely reversible airflow obstruction with a postbronchodilator FEVI/FVC $\geq 70 \%$ AHR became described if there has been $\mathrm{a} \geq 15 \% \mathrm{FEV} 1$ fall from baseline after inhalation of $4.5 \%$ hypertonic saline. An increase in postbronchodilator FEV $1 \geq 200 \mathrm{ml}$ and $12 \%$ in comparison with pre-bronchodilator FEV 1 was described as BDR. ${ }^{5}$ Patients with COPD had incompletely reversible airflow obstruction and post bronchodilator FEV 1 /FVC < 70\% and post-bronchodilator FEV $1<80 \%$ no AHR or BDR. Individuals with the asthma-COPD overlap had breathing signs and symptoms, extended airflow variability (asthma, i.e. AHR or BDR) in addition to incompletely reversible airflow obstruction (COPD; post-bronchodilator FEV 1 /FVC $<70 \%$ and post-bronchodilator FEV $1<80 \%$ of predicted).

\section{Participants}

Hundred thirty patients (130) in the age group of 40-80 years of both the genders were enrolled in this study from 20132015. Patents with history of suspected obstructive airway disease have been enrolled in this study in the outpatient department of SKIMS clinical. Suspected patients enrolled in this study have been subjected to spirometry. Patients found to have an obstructive pattern $(\mathrm{FEV} 1 / \mathrm{FVC}<0.7)$ have been enrolled in this study. These patients have been subjected to other investigations like CBC, sputum for eosinophils. Patients were additionally given a questionnaire concerning the age of onset of symptom, history of smoking, the number of exacerbations at some stage in the last 365 days (one year).

\section{RESULTS}

We analyzed the information gathered from the enrolled 130 patients for the prevalence of ACOS. Results display that the percentage of patients with asthma COPD overlap syndrome changed into $17.69 \%$ (23 patients), the percentage of patients with asthma only was $36.9 \%$ (48 patients) and the percentage of patients with COPD was $48.3 \%$ (59 patients) (Figure 1).

We further analyzed the patients with ACOS for history of smoking, it was observed that smoking had a significant effect on the prevalence of ACOS as $87 \%$ of patients with ACOS were having a history of smoking $(p<.001)$ (Figure.2).

Next, we analyzed the ACOS patients for the frequency of acute exacerbations, it was observed that the frequency of exacerbation's in ACOS patients was significantly higher 


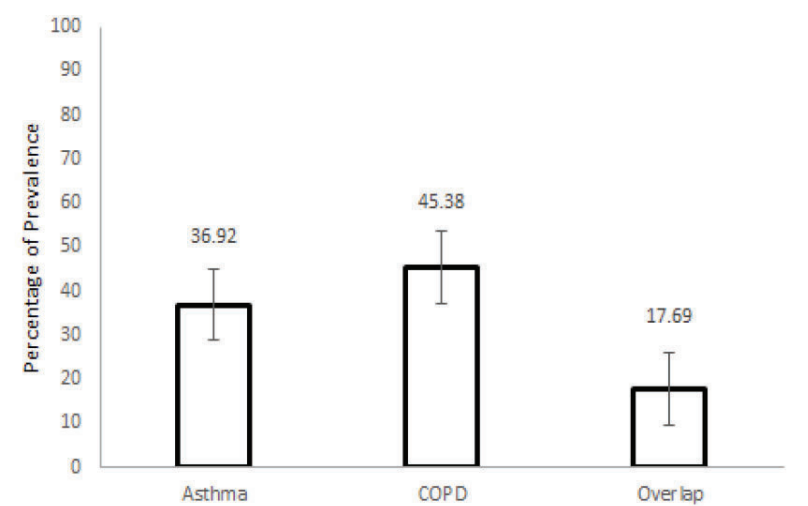

Figure-1: Percentage of the different phenotypes. The vertical lines on the bars represent standard error bars.

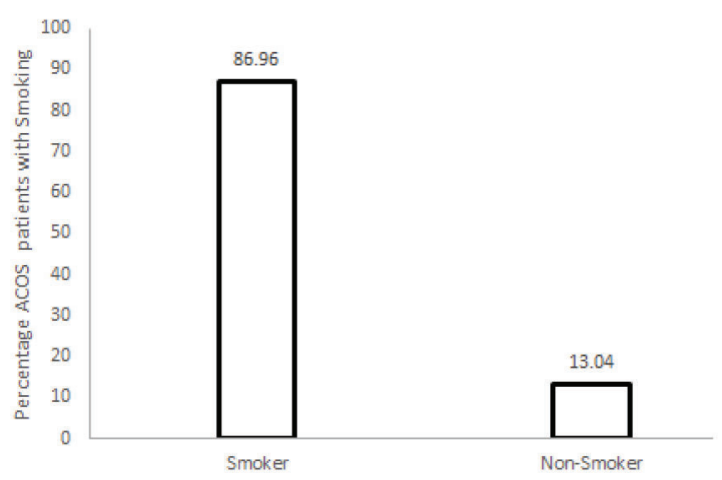

Figure-2: Percentage of ACOS patients with Smoking History

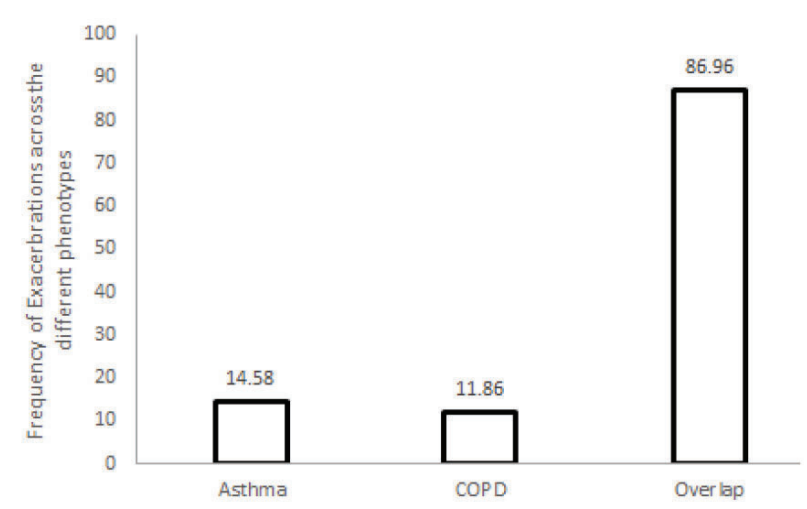

Figure-3: The frequency of exacerbations across the different phenotypes

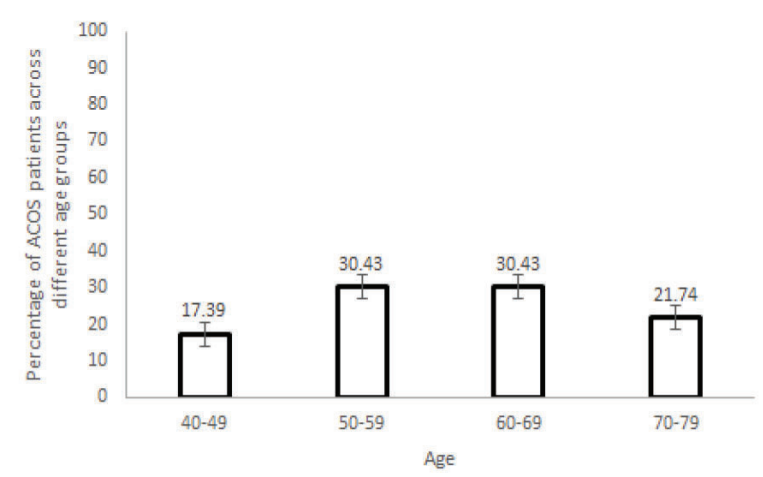

Figure-4: Percentage of ACOS patients across different age groups. The vertical lines on each bar represent standard error bars. compared to COPD ( $p$ <.001). Similarly the frequency of acute exacerbations in ACOS is significantly higher compared to asthma $(\mathrm{p}<.001)$. On comparing the frequency of exacerbations in ACOS with COPD we observed that the difference is not significant $(p>.05)$ (figure 3 ).

Furthermore, we investigated the prevalence of ACOS across different age groups, although the difference in the percentage of ACOS patients across different age groups in not significant however we observed that ACOS increases progressively with age (figure-4).

\section{DISCUSSION}

Allergies-COPD overlap syndrome is one of the phenotypes of obstructive airway ailment. It shares some features of asthma and few features of COPD. However, clinical scenario is extra complex and we regularly have cases having positive degree of overlapping. Some asthma patients smoke and have characteristics much like COPD with extra neutrophilic inflammation and increased lung function decline and decreased reaction to bronchodilators or corticosteroids. Similarly, we see COPD patients who present characteristics which might be traditionally attributable to asthma such as reversibility in bronchodilator response (BDR) and eosinophils in sputum. ${ }^{3}$ ACOS is poorly diagnosed in part because medical trials have constantly left out this phenotype, as evidenced with the aid of strict inclusion and exclusion standards that exclude either asthma patients from COPD patients or COPD patients from asthma patients. ${ }^{5}$ Asthmatic bronchitis was the term used to explain the overlapping situation of asthma and COPD by American Thoracic Society (ATS) in 1962, however no efforts were made to expound on this scientific phenotype till currently. ${ }^{5}$ The prevalence of ACOS increases with age. ${ }^{13}$ The presence of airway hyper responsiveness in COPD subjects leads to increased exacerbations. ${ }^{20}$ Patients with ACOS have a higher chance of exacerbation and hospitalization than people with COPD, have extra respiratory signs such as dyspnea and wheezing and decreased physical activity in comparison with COPD alone. ${ }^{21}$ ACOS patients consume 2 to 6-fold greater healthcare facilities as compared to asthma or COPD patients. ${ }^{14}$ By characterization and better understanding of ACOS can enable us to identify a group of patients of COPD who show a good response to inhaled corticosteroids in contrast to most patients with COPD, who have neutrophilic infiltration and does not respond to corticosteroids. ${ }^{25}$

The first part of our study was to investigate the prevalence of asthma-COPD overlap syndrome. Our study employed one hundred thirty stable patients of obstructive airway disease, which were assessed for the presence of asthma-COPD overlap syndrome, according to the standards given by GES EPOC Spanish guidelines. Out of one hundred thirty patients, 23 patients $(17.7 \%)$ were found to have asthmaCOPD overlap phenotype, 48 patients $(37 \%)$ were asthmatic and 59 patients $(45 \%)$ were COPD patients.

In our study we have found that the prevalence of ACOS is $17.7 \%$. Zeki et al., in their study have reported that the prevalence of asthma-COPD overlap syndrome varies between $15 \%$ to $25 \%$ in the adult population with obstructive 
airway disease $\mathrm{e}^{2}$, and our study is consistent with their finding. Hardin et al. in a recent cross-sectional study which included 915 subjects who have been labelled as COPD (GOLD stage 2-4). Out of 915 patients, 796(87\%) were labeled as having COPD alone. This study has slightly decreased incidence of ACOS in comparison with our study. This can be due to the fact this study has taken only COPD patients into consideration whereas our study has taken all patients with obstructive airway disease, which encompass both COPD asthma patients. Miravitlles et al. in their study (EPI-SCAN) observed that $17.4 \%$ of patients were having asthma-COPD overlap syndrome. ${ }^{4}$ Wei-Sheng Chung et al. had performed a study to compare the occurrence of acute respiratory events in ACOS and COPD cohorts in Taiwan. This study has reported that the prevalence of ACOS is $17.4 \%$ in patients with COPD alone. ${ }^{26}$ The overall prevalence of ACOS was about $17.4 \%$ in patients with COPD. Gibson et al. in a study on 44 older (adults aged $\geq 55$ years) adults with stable obstructive airway disease found that $65 \%$ of patients exhibited an overlap syndrome. ${ }^{3}$ Jung Wha Chung et al., in a population-based study including 9104 patients have shown that the prevalence of asthma-COPD overlap syndrome is $14.2 \%$. All these studies have shown results that are consistent

with the results in our study except the results shown by Gibson et al, where the prevalence of asthma- COPD overlap syndrome is higher $(65 \%)$. This variation in the prevalence of Asthma-COPD overlap in the study conducted by Gibson et al may be due to a smaller sample size (only 44 patients) and or an older age group (only $>55$ years older patients were included).

The second part of our study was designed to assess the demographic and clinical profile of patients with asthmaCOPD overlap syndrome. We additionally located that a few of the patients of ACOS only 8 (34.8\%) have been below 50 years of age and $15(65.2 \%)$ were above 50 years of age, depicting that prevalence of ACOS increases with age. However this is not statistically good sized p-value 0.457 . We also analyzed that the prevalence of asthma COPD overlap syndrome increases with age up to 60 years being $17.4 \%, 30.4 \%, 30.4 \%, 21.7 \%$ among 40-50, 50-60, 60-70, $70-80$ years of age respectively. We additionally analyzed that the prevalence of asthma increases with age up to 60 years, after 60 years the prevalence of asthma decreases. Our study also reveals that the prevalence of COPD progressively increases with age. Hardin et al in their study which included 915 patients showed that subjects with COPD and Asthma (overlap) were younger than those with COPD ( $\mathrm{p}$-value $0.0001) .{ }^{27}$ This is consistent with the results shown by our study although $\mathrm{p}$-value in our study is not statistically significant.

We observed that patients with bronchial asthma-COPD overlap have more common exacerbations ( $p$-value $<0.0001$ ). Menzes et al., of their study determined that patients with bronchial asthma-COPD overlap phenotype had been at higher chance for exacerbations (OR=4.11; 95\% CI: 1.45 11.67) compared to COPD patients. Hardin et al. observed that patients with asthma-COPD overlap phenotype have more frequent exacerbations $(\mathrm{P}$-value $<0.001) .{ }^{27}$ Wei Shung
Chung et al., found that patients with asthma-COPD overlap phenotype have frequent exacerbations $(\mathrm{OR}=2.58$ 95\% CI: 2.43-2.74). ${ }^{26}$

We observed that the majority of our asthma-COPD overlap patients are people who smoke ( $\mathrm{p}$-value $<0.008)$. Hardin et al in their study on 915 patients have shown that patients with asthma COPD overlap have fewer pack years in comparison to COPD alone patients. ${ }^{27}$ These all research have shown that asthma COPD overlap syndrome is related to smoking and the outcomes of these studies are consistent with the observations of the study that we conducted.

In Our study, we've proven that the prevalence of asthmaCOPD overlap syndrome does not change with the gender of the patient ( $p$-value 0.136 ). Hardin et al. in their study have shown that the prevalence of asthma-COPD overlap syndrome does not vary with the gender of the patient. ${ }^{27}$ Wei Shung Chung et al in their study has shown that the prevalence of asthma-COPD overlap syndrome is more in males as compared to females (56.9\% vs. $43.1 \%) .{ }^{26}$

We also observed in our study that the prevalence of Asthma-COPD overlap syndrome does not vary with the geographical distribution. Though in our study 11 patients of Asthma-COPD overlap syndrome were from rural areas and 12 patients were from urban areas but this is not statistically significant ( $p$-value 0.964). Currently, there are no studies available to show the relationship of asthma-COPD overlap syndrome with geographical distribution.

\section{CONCLUSION}

Asthma-COPD overlap syndrome represents a significant proportion among patients with obstructive airway disease in Kashmiri population. This group of patients has more frequent exacerbations and hence hospitalization. Consequently it is crucial to understand this group of patients due to the fact that management is different from the patients with COPD alone and institution of proper treatment in this group of patients can prevent frequent exacerbations and therefore hospitalizations, thereby lowering the burden on hospitals and other healthcare facilities.

\section{ACKNOWLEDGMENT}

The authors thank Zahid Maqbool for technical assistance and preparing the manuscript.

\section{REFERENCES}

1. Lange P, Partner J, Vestbo J, et al. A 15-year follow-up study of ventilatory function in adults with asthma. N Engl J Med 1998; 339(3):1194-2000.

2. Ray A, Cohn L. Th2 cells, and GATA-3 in asthma: new insights into the regulation of airway inflammation. J Clin Invest 1999; 104(1):985-93.

3. Gibson PG, Simpson JL. The overlap syndrome of asthma and COPD: what are its features and how important is it? Thorax 2009;64(8), 728-735.

4. Miravitlles M, calle Myriam, Ancochea J, Soriano JB et al Spanish COPD Guidelines (GesEPOC): Treatment of stable COPD. Arch Bronconeumol 2012; 48(7):247257.

5. Committee on Diagnostic Standards for Nontuberculous 
Respiratory Diseases. American Thoracic Society, 1962. Definitions and classification of chronic bronchitis, asthma, and pulmonary emphysema. Am. Rev. Respir. Dis. 1962; 85(3):762-769.

6. Zeki A. A, Michael Schivo, Andrew Chan, Timothy E. Albertson and Samuel Louie. The AsthmaCOPD Overlap Syndrome: Pharmacotherapeutic considerations; Expert Rev Clin Pharmacol. 2013;6(2):197-219.

7. Soler-CataluñaJJ, Cosío B,Izquierdo JL et al. Consensus document on the overlap phenotype COPD-asthma in COPD. Arch. Bronconeumol 2012;48(9):331-337.

8. Peces-Barba G, Barberà JA, Agustí A, Casanova C, Casas A, Izquierdo JL, et al. Guía Clínica SEPARALAT de diagnóstico y tratamiento de la EPOC. Arch Bronconeumol 2008;44(2):271-81.

9. GEMA 2009. Guía Espa nola para el Manejo del Asma. Madrid: Luzan (2009).

10. Tashkin DP, Celli B, Decramer M, Liu D, Burkhart $\mathrm{D}$, Cassino $\mathrm{C}$, et al. Bronchodilator responsiveness in patients with COPD. Eur Respir J 2008;31(6):742-50.

11. Han MK, Agustí A, Calverley PM, Celli BR, Criner G, Curtis JL, et al. Chronic obstructive pulmonary disease phenotypes. The future of COPD. Am J Respir Crit Care Med. 2010;182(3):598-604.

12. J. B. Soriano, K. J. Davis, B. Coleman, G. Visick, D. Mannino, and N. B. Pride. The proportional venn diagram of obstructive lung disease: two approximations from the United States and the United Kingdom. Chest, 2003; 124(5):474-481.

13. F. T. Shaya, D. Dongyi, M. O. Akazawa et al. Burden of concomitant asthma and COPD in a Medicaid population. Chest 2008;134(5):14-19.

14. K. M. Beeh, O. Kornmann, J. Beier, M. Ksoll, and R. Buhl, "Clinical application of a simple questionnaire for the differentiation of asthma and chronic obstructive pulmonary disease," Respiratory Medicine 2004;98(2):591-597.

15. Zeki A A, Michael Schivo, Andrew Chan, Timothy E. Albertson and Samuel Louie. The Asthma-COPD Overlap Syndrome: A Common Clinical Problem in the Elderly. J.allergy (cairo), 861926(2011)

16. Gurrera S. overlap of asthma and chronic obustructive pulmonary disease. Curr Opin Plum Med. 2005; 11(1): 7-13.

17. Hospers JJ, Postma DS, Rijcken B, Weiss ST, Schouten JP. Histamine airway hyperresponsiveness and mortality from chronic obstructive pulmonary disease: a cohort study. Lancet. 2000;356(6):1313-7.

18. Vestbo J, Edwards LD, Scanlon PD, Yates JC, Agusti A, Bakke P, et al. Changes in forced expiratory volume in 1 second over time in COPD. N Engl J Med. 2011;365(6):1184-92.

19. Siva R, Green RH, Brightling CE, Shelley M, Hargadon B, Mckenna W, et al. Eosinophilic airway inflammation and exacerbations of COPD: a randomized controlled trial. Eur Respir J. 2007;29(3):906-13.

20. Grupo de trabajo de GESEPOC. Hacia un nuevo enfoque en el tratamiento de la EPOC. Guía Espa nola de la EPOC (GESEPOC). Arch Bronconeumol. 2011;47(1):379-81.
21. J. Elias. The relationship between asthma and COPD: lessons from transgenic mice. Chest, 2004; 126(2): 111S-116S.

22. Gibson PG, Girgis-Gabardo A, Morris MM, et al. Cellular characteristics of sputum from patients with asthma and chronic bronchitis. Thorax 1989; 44(3):693699.

23. Kanazawa H, Shoji S, Yamada M, et al. Increased levels of nitric oxide derivatives in induced sputum in patients with asthma. J Allergy Clin Immunol 1997; 99(2):624629.

24. Fahy JV, Boushey HA, Lazarus SC, et al. Safety and reproducibility of sputum induction in asthmatic subjects in a multicenter study. Am J Respir Crit Care Med 2001; 163(6): 1470-1475

25. Decramer M, Janssens W, Miravitlles M. Chronic obstructive pulmonary disease. Lancet 2012; 379(5):1341-1347

26. Wei-Sheng Chung, Cheng-Li Lin, and ChiaHuComparison of Acute Respiratory Events Between Asthma-COPD Overlap Syndrome and COPD Patientsng Kao. Volume 94, Number 17, May 2015

27. Silverman EK, Hardin M, Barr RG, Hansel NN, Schroeder JD, Make BJ, et al. The clinical features of the overlap between COPD and asthma. Respir Res. 2011;12(5):127.

\section{Source of Support: Nil; Conflict of Interest: None}

Submitted: 01-02-2019; Accepted: 02-03-2019; Published online: 13-03-2019 